\title{
Analysis of clinical features and prognosis of malignant triton tumor: A report of two cases and literature review
}

\author{
GUO LI ${ }^{1,2}$, CHAO LIU ${ }^{1,2}$, YONG LIU ${ }^{1,2}$, FANG XU $^{1,2}$, ZHONGWU SU $^{1,2}$, YUNYUN WANG $^{1,2}$, SHULING REN $^{1,2}$, \\ TENGBO DENG ${ }^{1,2}$, DONGHAI HUANG ${ }^{1,2}$, YONGQUAN TIAN ${ }^{1,2}$ and YUANZHENG QIU ${ }^{1,2}$
}

${ }^{1}$ Department of Otolaryngology and Head-Neck Surgery, ${ }^{2}$ Otolaryngology Major Disease Research Key Laboratory of Hunan, Xiangya Hospital, Central South University, Changsha, Hunan 410008, P.R. China

Received September 28, 2014; Accepted June 22, 2015

DOI: $10.3892 / 01.2015 .3762$

\begin{abstract}
Malignant triton tumor(MTT) is a malignant peripheral nerve sheath tumor with rhabdomyoblastic differentiation. The prognosis of patients is poor, and due to its rarity, large case studies are lacking. The aim of this study is to describe the clinical features and identify potential prognostic factors. Two patients with MTT in the head and neck treated at our department are reported. A literature search revealed another 198 published cases. All of these cases then went through a retrospective analysis. The ratio of male-to-female incidence was $1.5: 1$, and the median age at diagnosis was 29 years. In $41.7 \%$ of cases it occurred in patients with neurofibromatosis type 1 . The five-year survival of MTT was found to be just $35 \%$. Cox proportional hazards analysis revealed that complete resection (hazard ratio, $0.396 ; \mathrm{P}=0.032$ ) and metastases (hazard ratio, 3.188; $\mathrm{P}=0.004$ ) were associated with mortality, indicating that complete resection may lead to a longer life span, and that the existence of metastasis suggested a worse prognosis for patients with MTT.
\end{abstract}

\section{Introduction}

Malignant triton tumor (MTT) is a malignant peripheral nerve sheath tumor (MPNST) with rhabdomyoblastic differentiation $(1,2)$. The name 'triton' derived from an experiment in which sciatic nerve tissue of the triton salamander was stimulated to grow a neoplasm with skeletal muscle components (3). It was first described as a disease in 1932 (4) and first reported as 'malignant triton tumor' in 1973 (5). Woodruff et al proposed three criteria for its diagnosis (5): i) arising along a peripheral nerve in ganglioneuroma or in a patient with neurofibromatosis type $1(\mathrm{NF}-1)$ or representing a metastasis from such a

Correspondence to: Professor Yuanzheng Qiu, Department of Otolaryngology and Head-Neck Surgery, Xiangya Hospital, Central South University, 87 Xiangya Road, Changsha, Hunan 410008, P.R. China

E-mail: xyqyz@hotmail.com

Key words: malignant triton tumor, surgery, prognosis, survival analyses tumor; ii) having the growth characteristics of a Schwann cell tumor; and iii) rhabdomyoblasts arise within the body of the tumor. Daimaru et al considered that sporadic cases fulfilling the latter two criteria could be diagnosed (6). Clinically, the morbidity of MTT is less than 1 per 1,000,000 (7). As it is a rare tumor, only limited cases have been reported so far, and no large group studies exist. For further understanding of the clinical features of MTT, we share our experiences of the treatment of MTT in our hospital and conduct a literature analysis. Here, we review two cases of MTT occurring in the head and neck, with no evidence or symptoms of NF-1. Written informed consent was obtained from both patients and the study was approved by the Research Ethics Committee of Central South University (Changsha, China).

\section{Case report}

Case 1. A 27-year-old male was admitted to Xiangya Hospital, Changsha, China, in March 2007, complaining of pain and a foreign body sensation in the left side of the pharynx. Physical examination revealed a cauliflower-like brown mass $(30 \times 20 \mathrm{~mm})$ covered with grayish membrane at the left tonsillar fossa. It infiltrated into the surrounding tissues. The patient's mouth opened freely and no lymphadenopathy was observed. Magnetic resonance imaging (MRI) revealed isodense T1 and long T2 signals, and there was no overt submaxillary bony destruction (Fig. 1A-C). On April 5, 2007, the patient underwent ligation of the external carotid artery via a combined jaw-and-neck approach and tumor resection en bloc via a combined parapharyngeal space and intra-oral approach. The surrounding tissues were invaded and the tumor had adhered too tightly to be separated. The resected tissues included partial mucosa of the posterior pharyngeal wall and left fauces, partial medial and lateral pterygoid muscle and the left submandibular gland en bloc. The histological diagnosis was MTT with positive myogenin and S-100. The marginal mandibular branch was preserved. Following an uneventful postoperative recovery, the patient was discharged 7 days after surgery.

Following a course of radiotherapy at a total dose of 52 Gy in May 2007, two courses of chemotherapy with combined pirarubicin, ifosfamide and carboplatin were administered. The patient underwent partial mandibulectomy for 
osteonecrosis in 2008 and was treated for granulocytopenia following chemotherapy. Follow-up was carried out until April 2014. There was no evidence of further disease.

Case 2. A 43-month-old boy presented with hoarseness for 10 days, failure to swallow saliva for 5 days and headache and vomiting for 2 days. Clinical and radiological examinations revealed a mass of $\sim 2.2 \times 2.8 \times 2.5 \mathrm{~cm}$ in the right jugular foramen area with destruction of the skull base. MRI demonstrated isodense T1 and long T2 signals, while gadolinium-diethylenetriamine pentaacetic acid-enhanced MRI indicated enhancement of the mass with a distinct lobulated margin (Fig. 1D-F). Surgery revealed that the neoplasm was located behind the temporomandibular joint and had destroyed the temporal bone and skull base. With an invasion of the deep paroid, the neoplasm had a tight connection with the internal jugular vein. Rapid intraoperative pathological diagnosis suggested a spindle cell sarcoma with an inclination of MPNST. At the request of his parents, surgery was not performed and no treatment was offered. Hematoxylin and eosin staining and immunohistochemistry results suggested MTT with Bcl-2(+), CD34(+), CD57(-), CD99(+), desmin(+), HMB45(-), myogenin(++), NF(+), vimentin(+) and S-100(-) (Fig. 2). The child then survived only seven months.

\section{Literature review}

Database analysis. Literature searches on PubMed were conducted with the terms 'malignant triton tumor' and 'malignant peripheral nerve sheath tumors AND rhabdomyosarcoma' for the period prior to April 2014. All MTT cases were extracted from the databases. The relevant information included standard demographic information (age, gender and year of diagnosis) as well as NF-1, onset location, surgical approach, type of adjuvant therapy (radiotherapy and chemotherapy), relapse and metastasis. Survival time and final status were also recorded and the follow-up period was designated to be 60 months.

The onset location of MTT was mainly classified as trunk, head/neck or limbs, according to the original location. Where the tumor occurred in more than two places without a known original location, it was defined as 'multiple locations'. The concept of complete resection refers to radical resection, amputation or en bloc resection with microscopically negative margins. The data on adjuvant radiotherapy and chemotherapy were recorded as dichotomous variables. Local recurrence was defined as any tumor with complete resection which redeveloped in the surgical excision bed. Metastasis was defined as any distant tumor spread which was confirmed via biopsy or diagnosed with clinical experience, such as positron emission tomography-computed tomography (PET-CT) or radionuclide bone imaging. One patient who succumbed during surgery was also excluded from our analysis. The data that was not available from the literature was classified as 'Unknown'.

All the data were imported into SPSS version 18.0 software (SPSS Inc, Chicago, IL, USA). Standard descriptive statistics were computed for those selected variables. Kaplan-Meier survival analysis was performed for the whole cohort. The potential effects of clinical variables on survival were determined by the Cox proportional hazards model. All variables
Table I. Onset location of malignant triton tumors.

\begin{tabular}{llc}
\hline Onset location & \multicolumn{1}{c}{$\begin{array}{c}\text { Onset location } \\
\text { in detail }\end{array}$} & $\begin{array}{c}\text { Cases, } \mathrm{n} \\
(\%)\end{array}$ \\
\hline Trunk & Pelvic cavity & $12(6.0)$ \\
& Mediastinum & $12(6.0)$ \\
& Spine & $10(5.0)$ \\
& Retroperitoneal space & $9(4.5)$ \\
& Chest wall & $8(4.0)$ \\
& Buttocks & $5(2.5)$ \\
& Lung & $5(2.5)$ \\
Head and neck & Back & $3(1.5)$ \\
& Other & $12(6.0)$ \\
& Total & $76(38.0)$ \\
& Nasal cavity and sinus & $20(10.0)$ \\
& Neck & $19(9.5)$ \\
& Brain & $11(5.5)$ \\
& Oral cavity and around & $9(4.5)$ \\
& Parapharyngeal space & $4(2.0)$ \\
Limbs & Occiput & $3(1.5)$ \\
& Other & $7(3.5)$ \\
Onknown & Total & $73(36.5)$ \\
& Legs & $35(17.5)$ \\
& Arm & $7(3.5)$ \\
& Palm & $1(0.5)$ \\
& Foot & $1(0.5)$ \\
& Total & $44(22.0)$ \\
& Total & $3(1.5)$ \\
& & $4(2.0)$ \\
& & $200(100.0)$ \\
& &
\end{tabular}

were entered simultaneously into a multivariate model. $\mathrm{P}<0.05$ was considered to indicate a statistically significant difference.

Demographic and clinical data of MTT. A total of 198 cases were retrieved from 135 studies related to MTT in the databases. Pooling the data of our cases, the data of 200 MTT cases were collected in total. The male-to-female ratio was 1.5 to 1 . The median age at diagnosis was 29 years old. The age group distribution chart revealed that the patients with NF-1 were most common in the 20-39 age groups while patients without NF-1 had two peak age groups: the 20s and 50s (Fig. 3A). A total of $41.7 \%$ of patients had a history of NF-1 and the others were sporadic. Among all patients, the affected sites included the trunk $(n=76,38.0 \%)$, head and neck $(n=73,36.5 \%)$ and extremities $(n=44,22.0 \%$; Table I). The clinical treatment data revealed that $68.7 \%$ of tumors were removed completely. Radiotherapy and chemotherapy were administered to $54.0 \%$ and $35.3 \%$ of patients, respectively. The rates of recurrence and metastasis were $42.6 \%$ and $34.4 \%$, respectively.

Analysis of prognostic factors of MTT. A total of 133 cases with follow-up were selected for the prognosis analysis. Life 
Table II. Kaplan-Meier analysis and Cox proportional hazards analyses of patients with malignant triton tumor.

\begin{tabular}{|c|c|c|c|c|c|c|}
\hline \multirow[b]{2}{*}{ Category } & \multicolumn{3}{|c|}{ Kaplan-Meier analysis } & \multicolumn{3}{|c|}{ Cox proportional hazards analyses } \\
\hline & Number $^{\mathrm{a}}$ & $\begin{array}{l}\text { Mean time to } \\
\text { death, months }\end{array}$ & P-value & HR & $95 \%$ CI for HR & P-value \\
\hline Gender & & & 0.207 & & & 0.494 \\
\hline Male & 78 & 34.24 & & & & \\
\hline Female & 53 & 28.46 & & 1.312 & $0.602-2.858$ & \\
\hline \multicolumn{7}{|l|}{ Age, years ${ }^{c}$} \\
\hline$\leq 32$ & 68 & 25.68 & $0.013^{\mathrm{d}}$ & & & 0.423 \\
\hline$>32$ & 65 & 36.87 & & 0.736 & $0.602-1.558$ & \\
\hline Location & & & 0.638 & & & \\
\hline Head and neck & 48 & 29.22 & & & & \\
\hline Trunk & 56 & 31.87 & & 0.944 & $0.392-2.272$ & 0.845 \\
\hline Limbs & 26 & 30.37 & & 0.912 & $0.602-2.303$ & 0.942 \\
\hline NF-1 & & & $0.000^{\mathrm{d}}$ & & & 0.440 \\
\hline Yes & 72 & 17.80 & & & & \\
\hline No & 40 & 36.59 & & 1.312 & $0.628-2.914$ & \\
\hline Complete resection & & & $0.010^{\mathrm{d}}$ & & & $0.032^{\mathrm{d}}$ \\
\hline Yes & 76 & 34.23 & & & & \\
\hline No & 39 & 22.50 & & 0.396 & $0.170-0.925$ & \\
\hline Radiotherapy & & & $0.002^{\mathrm{d}}$ & & & 0.131 \\
\hline Yes & 67 & 36.24 & & & & \\
\hline No & 54 & 22.99 & & 0.572 & $0.277-1.181$ & \\
\hline Chemotherapy & & & 0.430 & & & 0.244 \\
\hline Yes & 41 & 31.97 & & & & \\
\hline No & 80 & 29.15 & & 0.594 & $0.248-1.426$ & \\
\hline Recurrence & & & 0.129 & & & 0.511 \\
\hline Yes & 45 & 27.82 & & & & \\
\hline No & 66 & 35.96 & & 1.278 & $0.615-2.655$ & \\
\hline Metastasis & & & $0.000^{\mathrm{d}}$ & & & $0.004^{\mathrm{d}}$ \\
\hline Yes & 37 & 18.12 & & & & \\
\hline No & 71 & 39.80 & & 3.188 & $1.450-7.008$ & \\
\hline
\end{tabular}

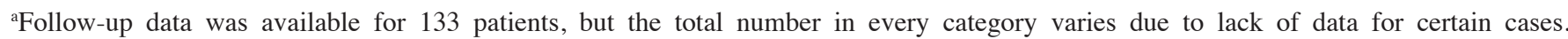
${ }^{b}$ Estimation is limited to the longest survival time if censored. ${ }^{c}$ The median age of the 133 patients with follow-up data was 32 years. ${ }^{d} \mathrm{P}<0.05$. HR, hazard ratio; CI, confidence interval.

span analysis revealed that the 1-, 2- and 5-year survival rates were $69 \%, 48 \%$ and $35 \%$, respectively (Fig. 3B). The results of Kaplan-Meier survival analysis demonstrated that five factors had a statistically significant effect on the survival curves; namely, age $(\mathrm{P}=0.013)$, presence of $\mathrm{NF}-1(\mathrm{P}<0.001)$, complete resection $(\mathrm{P}=0.010$, Fig. $3 \mathrm{C})$, radiotherapy $(\mathrm{P}=0.002)$ and presence of metastasis $(\mathrm{P}<0.001$, Fig. 3D). The factors of gender, onset site, chemotherapy and relapse had no significant effect on the survival curves ( $\mathrm{P}>0.05$, Table II).

On this basis, all factors in the Kaplan-Meier survival analysis were entered simultaneously into the Cox proportional hazards model for analysis. The result revealed that the factors of complete resection and metastasis had a significant effect on survival $(\mathrm{P}<0.05$, Table II). The hazard ratio $(\mathrm{HR})$ for the former was 0.396 . This indicated that if tumor excision was performed en bloc, there was a reduction in mortality risk. The HR of the latter was 3.188, implying that mortality risk increased more than three times.

\section{Discussion}

Although the mechanism of MTT remains elusive, its association with nerves or nervous lesions should be noted. The literature revealed that a number of nerves could be involved, including the cervical plexus (8), brachial plexus (9), optic nerve (10), sciatic nerve (11), cervical sympathetic nerve (12) and spinal nerve root (13). There was no conclusive evidence that the masses in our cases came from the nerves; however, the onset locations were observed to be in or around the parapharyngeal space which contains the nerves. A total of 50-70\% 


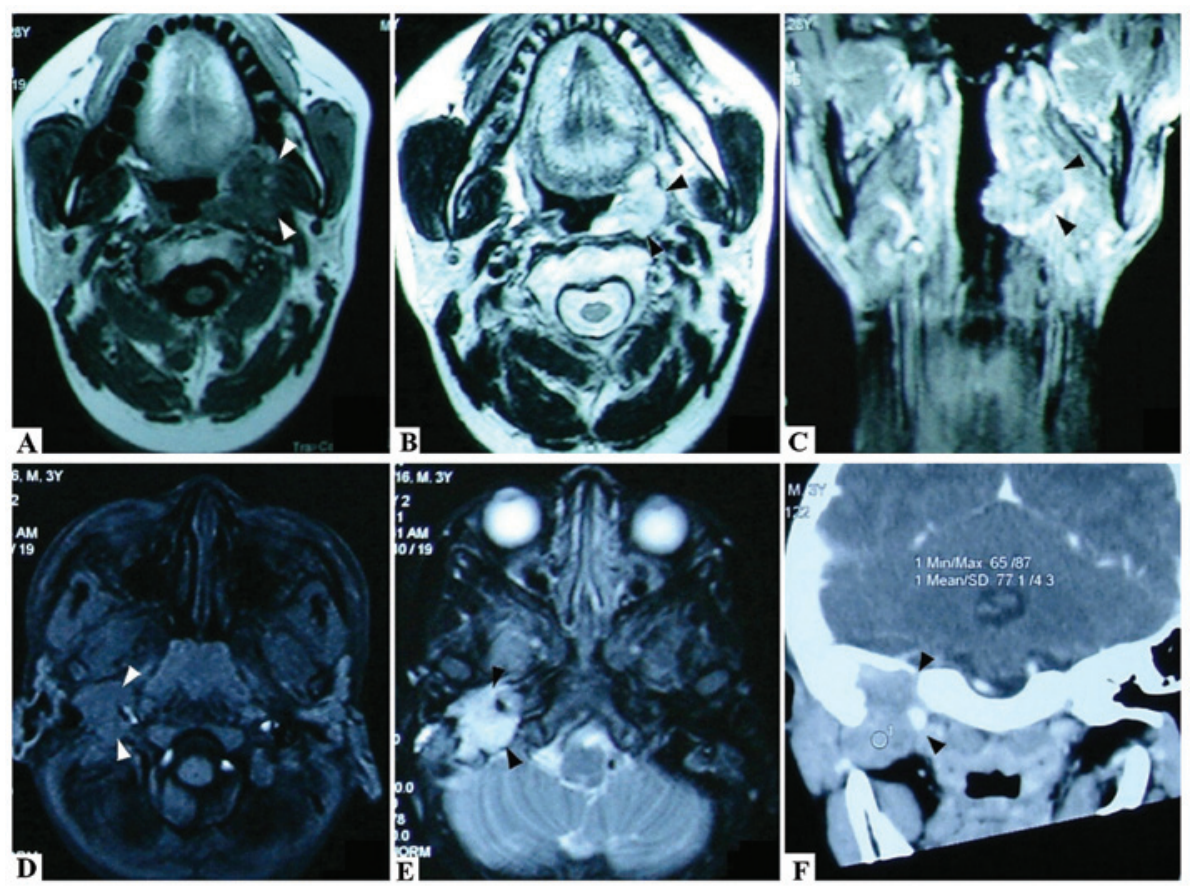

Figure 1. Magnetic resonance imaging (MRI) and computed tomography (CT) scan results of the malignant triton tumor (MTT) cases in our hospital (A) MRI results revealed that the mass had long or isodense T1 signals. (B and C) The mass demonstrated long T2 signals. In certain images, there are ring or linear forms, as a type of isolation strip, with isodense T2 signals, which are likely due to necrosis and hemorrhage in the mass. (D) MRI revealed isodense T1 signals in the tonsil MTT. (E) Gadolinium-diethylenetriamine pentaacetic acid-enhanced MRI indicated an enhancement of the mass with a distinct lobulated margin in the parapharyngeal space MTT. (F) Enhanced spiral CT scanning of the parapharyngeal space MTT.
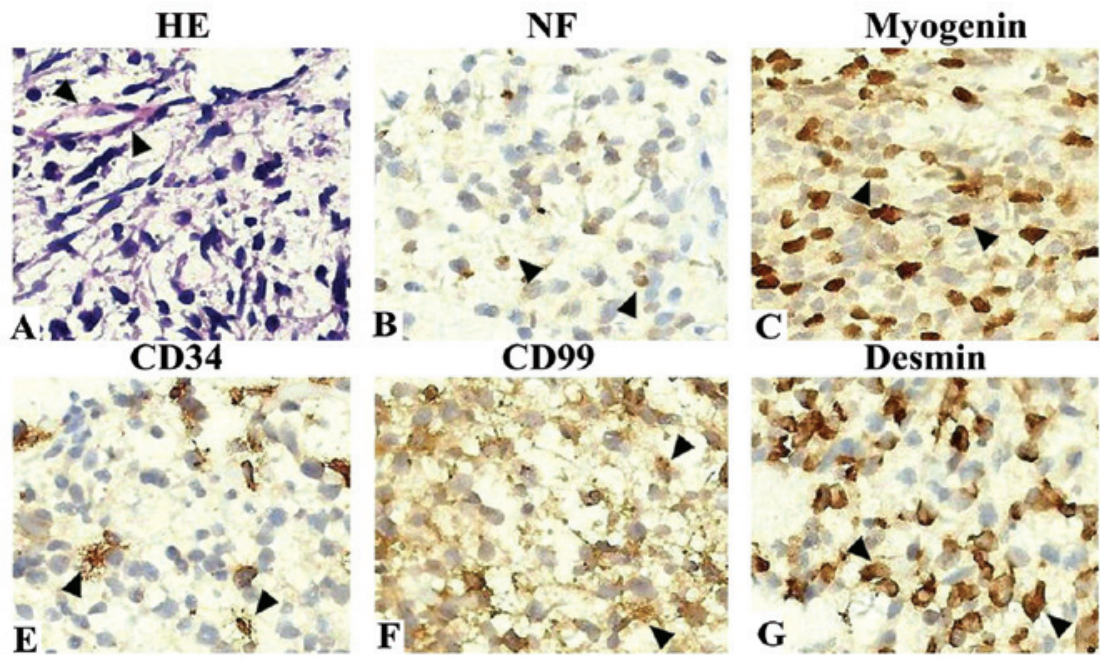

\section{Bcl-2}

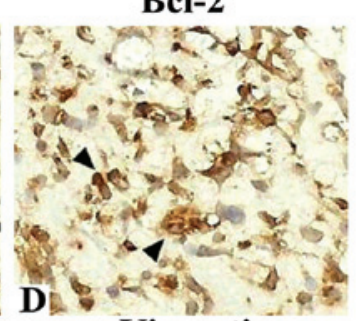

Vimentin

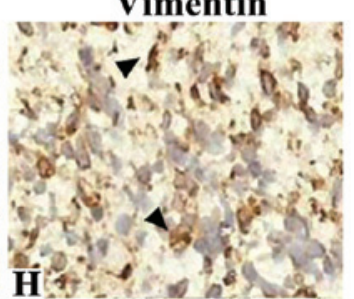

Figure 2. Images of hematoxylin and eosin (HE) staining and immunohistochemistry results of malignant triton tumor (MTT). (A) Images of HE staining. Large pleomorphic rhabdomyoblastic cells with abundant eosinophilic cytoplasm are embedded in a spindle cell tumor with a fine fibrillary matrix. (B-H) Immunohistochemistry results of MTT. The immunohistochemistry results were positive for $\mathrm{NF}(+), \mathrm{myogenin}(++), \mathrm{Bcl}-2(+), \mathrm{CD} 34(+), \mathrm{CD} 99(+)$, desmin(+) and vimentin(+). Magnification, x400.

of cases were observed to arise in patients with NF-1 $(1,7)$, and this rate was $\sim 40 \%$ in our own data. Radiotherapy and repeated surgery on the benign nerve fibroma may increase the risk of rhabdomyosarcoma differentiation $(11,14)$. At the sub-cellular level, aberrations in chromosomes 1, 6, 7, 8, 9, 16, 17, 19, 20 and 22 were correlated with the genesis and recurrence of MTT (15). The missense mutation and a loss of heterozygosity were observed in the majority of MTT cases $(16,17)$. Nucleotide deletion in P53, amplification of C-myc and aberrations in the hedgehog-patched pathway were also noted in MTT (18-20).
Clinically, the age at diagnosis age ranged from newborn to over 80 years old, with a median age of $29-35(1,21,22)$. More than $60 \%$ of MTT cases with NF-1 were diagnosed in patients between 20 and 50 years old, when the benign masses were suspected to undergo a malignant transformation (23). MTT was reported mainly in the head, neck and trunk, and less frequently in the extremities (1); our data yielded similar results. In the early stage, patients often had no self-reported symptoms, and only sought medical attention when there were such symptoms as pain or dysfunction caused by the 


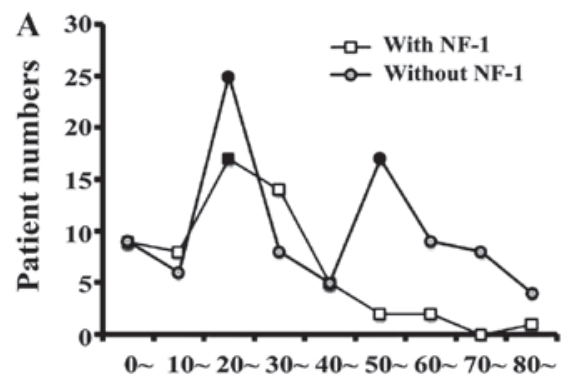

Age (year)

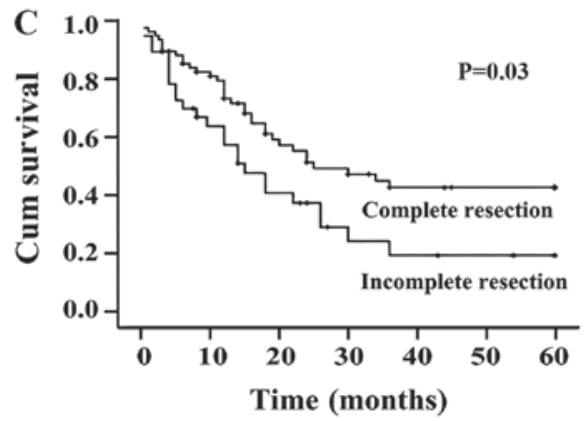

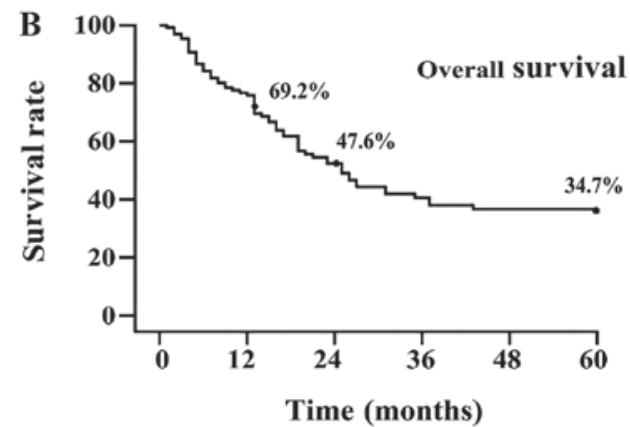

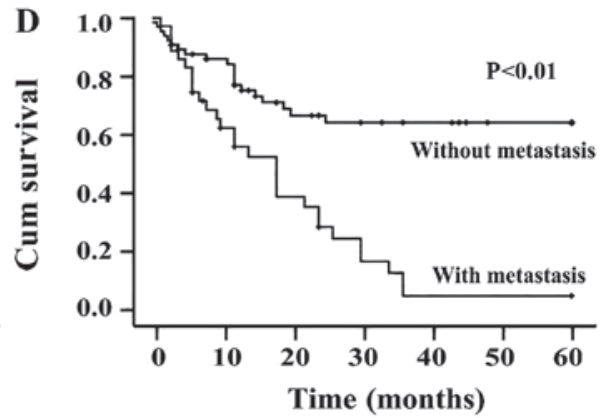

Figure 3. Age distribution and survival analysis of MTT patients. (A) Results revealed that patients with NF-1 were most common in the 20-39 age group while those without NF-1 had two peaks in the 20s and 50s. (B) Life span analysis revealed that the 1-, 2- and 5-year survival rates were $69 \%$, 48\% and 35\%, respectively. (C and D) Kaplan-Meier survival analysis of the data for the 133 cases with follow-up revealed that complete resection $(\mathrm{P}=0.010)$ and metastasis $(\mathrm{P}<0.001)$ had a statistically significant effect on the survival curves.

involvement with adjacent tissues. The size of the abdominal mass increased due to the lack of space constraints, and the maximal diameter was reported at $82 \mathrm{~cm}$ (24).

MRI has been the most common method of identifying the location, scale and shape of lesions. In most cases, the masses demonstrated short or mixed isodensity T1 and long T2 signals. In certain images, ring or linear forms were observed as a type of isolation strip; these are likely to be due to necrosis and hemorrhage in the mass as a result of a quick growth (25). Enhanced spiral CT scanning is capable of visualizing bone erosion around the mass and providing indications of its malignancy. 18FDG-PET-CT was also considered to be a more effective method of estimating the prognosis and metastasis (9). All of the above methods are used for auxiliary examination only, and the final diagnosis should be based upon pathology. Microscopically, MTT always contains shuttle-like malignant peripheral nerve cells, additional rhabdomyoblastic differentiation and zonal cytoplasm (7). Immunohistochemical results facilitated our diagnosis, particularly with positive desmin and myoglobin (13). Here, additional oncogene proteins including Bcl-2, CD34, CD99, HHF35, NF and vimentin were also noted to be positive, which confirmed the malignant features of this neoplasm.

Literature revealed that the 5-year survival rates of patients with MPNST and MTT were 34-44\% and 10-20\%, respectively $(3,4)$. Our data revealed 1-, 2- and 5-year survival rates of $69 \%, 48 \%$ and $35 \%$, respectively. Theoretically, the actual survival rates may be lower, since our censored proportion accounted for almost $20 \%$. Our analysis also revealed no survival difference among MTT of the trunk, head and neck, or extremities. Terzic et al observed that the 1-, 2- and 5-year survival rates of head and neck MTT were 76\%, 61\% and 49\%, respectively, and that paranasal sinus MTT had an even better prognosis (22).
The poor survival rate associated with distant metastasis was in keeping with the findings made in previous studies $(1,13)$.

In $\sim 60 \%$ of the cases in the literature, tumors were completely resected. Wide local excision, whole organ excision and amputation were performed $(7,22)$. In our analysis, Cox proportional hazards analysis demonstrated that complete resection is an independent prognostic factor, and decreased the mortality risk. Thus, radical mass excision is key to prolonging postoperative survival. The effectiveness of radiotherapy remains controversial. The single factor analysis of radiotherapy revealed a statistically significant difference; however, multi-factorial analysis revealed no difference, which may be related to the limited number of cases. Given our findings and experience, radiotherapy should be considered as a routine for all MTT patients, whether the residual mass remains or not. Ifosfamide, dactinomycin, carboplatin and fluorouracil are the main drugs used in chemotherapy $(4,22)$. One study described a tumor expressing retinoic acid receptors-a and $b$, and the treatment of isotretinoin and interferon- $\alpha$ was offered to control the disease (26). Kaplan-Meier analysis revealed no significant difference for the patients with chemotherapy, which might be attributed to the reason that there was no standard or guidelines. Simultaneously, the side effects of radiotherapy and chemotherapy should be recorded and managed promptly. Our patient (case 1) suffered osteonecrosis and granulocytopenia following adjunctive therapies. Thus, prolonging the survival time is not the only target for patients: improving their quality of life is also considered to be a priority.

\section{Acknowledgements}

Grants were obtained by the National Natural Science Foundation of China (nos. 81372426, 81372906, 81202128 and 
81172558), the Research Fund for the Doctoral Program of Higher Education of China (no. 20120162120049), the Graduate Student Research Innovation Project of Hunan Province (no. CX2013B108), the Freedom Explore Program of Central South University (no. 2012QNZT099), and the Open-End Fund for the Valuable and Precision Instruments of Central South University (no. CSUZC2014048).

The authors are grateful for the statistical guidance from Deng Jing at the Department of Statistics, School of Public Health, Central South University, and Zhu Lei at the Library of Medicine, Central South University, who offered assistance in the literature searches.

\section{References}

1. McConnell YJ and Giacomantonio CA: Malignant triton tumors complete surgical resection and adjuvant radiotherapy associated with improved survival. J Surg Oncol 106: 51-56, 2012.

2. Friesenbichler J, Leithner A, Maurer-Ertl W, Szkandera J, Sadoghi P, Frings A, Maier A, Andreou D, Windhager R and Tunn PU: Surgical therapy of primary malignant bone tumours and soft tissue sarcomas of the chest wall: a two-institutional experience. Int Orthop 38: 1235-1240, 2014.

3. Locatelli P: Formation de Membres Surnumeraires. C. R. Assoc. des Anatomistes, 20e reunion Turin, pp279-282, 1925.

4. Masson P: Recklinghausen's neurofibromatosis, sensory neuromas and motor neuromas. In: Libman Anniversary. Vol 2. International Press, New York, NY, pp793-802, 1932.

5. Woodruff JM, Chernik NL, Smith MC, Millett WB and Foote FW Jr: Peripheral nerve tumors with rhabdomyosarcomatous differentiation (malignant 'Triton' tumors). Cancer 32: 426-439, 1973.

6. Daimaru Y, Hashimoto H and Enjoji M: Malignant 'triton' tumors: a clinicopathologic and immunohistochemical study of nine cases. Hum Pathol 15: 768-778, 1984.

7. John M: Anderson's Pathology. The C. V. Mosby Company, Philadelphia, pp1894-1896, 1990.

8. Yang BB, Jiang $\mathrm{H}$ and Chang HY: Malignant triton tumour of the parapharyngeal space: a case arising from the cervical sympathetic nerve. J Laryngol Otol 122: 531-534, 2008.

9. Dartnell J, Pilling J, Ferner R, Cane P and Lang-Lazdunski L: Malignant triton tumor of the brachial plexus invading the left thoracic inlet: a rare differential diagnosis of pancoast tumor. J Thorac Oncol 4: 135-137, 2009.

10. Han DH, Kim DG, Chi JG, Park SH, Jung HW and Kim YG: Malignant triton tumor of the acoustic nerve. Case report. J Neurosurg 76: 874-877, 1992.

11. Tripathy K, Mallik R, Mishra A, Misra D, Rout N, Nayak P, Samantray S and Rath J: A rare malignant triton tumor. Case Rep Neurol 2: 69-73, 2010.
12. Cano JR, Algar FJ, Alvarez A and Salvatierra A: Triton tumor of the left sympathetic nerve. Interact Cardiovasc Thorac Surg 5: 790-791, 2006.

13. Rekhi B, Jambhekar NA, Puri A, Agrawal M and Chinoy RF: Clinicomorphologic features of a series of 10 cases of malignant triton tumors diagnosed over 10 years at a tertiary cancer hospital in Mumbai, India. Ann Diagn Pathol 12: 90-97, 2008.

14. Ozer E, Erkilic S, Bayazit YA, Mumbuc S, Aydin A and Kanlikama M: Malignant triton tumor of the supraclavicular region arising after radiotherapy. Auris Nasus Larynx 29: 405-407, 2002.

15. Koutsimpelas D, Brieger J, Heinrich U, Torzewski M, Sommer C and Mann WJ: Cytogenetic analysis of a malignant triton tumour by comparative genomic hybridization $(\mathrm{CGH})$ and review of the literature. Eur Arch Otorhinolaryngol 268: 1391-1396, 2011.

16. Strauss BL, Gutmann DH, Dehner LP, Hirbe A, Zhu X, Marley EF and Liapis H: Molecular analysis of malignant triton tumors. Hum Pathol 30: 984-988, 1999.

17. Ferrari A, Bisogno G, Macaluso A, Casanova M, D'Angelo P, Pierani P, Zanetti I, Alaggio R, Cecchetto $G$ and Carli M: Soft-tissue sarcomas in children and adolescents with neurofibromatosis type 1. Cancer 109: 1406-1412, 2007.

18. Chao MM, Levine JE, Ruiz RE, Kohlmann WK, Bower MA, Petty EM and Mody RJ: Malignant triton tumor in a patient with Li-Fraumeni syndrome and a novel TP53 mutation. Pediatr Blood Cancer 49: 1000-1004, 2007.

19. Haddadin MH, Hawkins AL, Long P, Morsberger LA, Depew D, Epstein JI and Griffin CA: Cytogenetic study of malignant triton tumor: A case report. Cancer Genet Cytogenet 144: 100-105, 2003.

20. Bura M, Musani V, Cretnik M, Botica I and Levanat S: Hedgehog-Patched pathway aberrations in a malignant triton tumor case study. Oncol Rep 20: 347-352, 2008.

21. Sönmez K, Türkyilmaz Z, Karabulut R, Kapisiz A, Eser EP, Memis L and Basaklar AC: A Triton tumor mimicking sacrococcygeal teratoma. J Pediatr Surg 44: e5-e8, 2009.

22. Terzic A, Bode B, Gratz KW and Stoeckli SJ: Prognostic factors for the malignant triton tumor of the head and neck. Head Neck 31: 679-688, 2009.

23. Duat-Rodríguez A, Carceller Lechón F, López Pino MÁ, Rodríguez Fernández $\mathrm{C}$ and González-Gutiérrez-Solana L: Neurofibromatosis type 1 associated with moyamoya syndrome in children. Pediatr Neurol 50: 96-98, 2014.

24. Murtaza B, Gondal ZI, Mehmood A, Shah SS, Abbasi MH, Tamimy MS and Kazmi ST: A huge malignant triton tumour. J Coll Physicians Surg Pak 15: 728-730, 2005.

25. Ghosh A, Sastri SB, Srinivas D, Mahadevan A, Anandappa CB and Shankar SK: Malignant triton tumor of cervical spine with hemorrhage. J Clin Neurosci 18: 721-723, 2011.

26. Köstler WJ, Amann G, Grunt TW, Singer CF, Schneider SM, Brodowicz T, Tomek S and Zielinski CC: Recurrent malignant Triton tumour: First report on a long time survivor. Oncol Rep 10: 533-535, 2003. 